\title{
Immunoglobulin $V_{H}$ somatic hypermutation in mantle cell lymphoma: mutated genotype correlates with better clinical outcome
}

\author{
Raymond Lai ${ }^{1}$, Shilo V Lefresne ${ }^{1}$, Bevin Franko ${ }^{1}$, David Hui ${ }^{2}$, Imran Mirza ${ }^{1}$, \\ Adnan Mansoor ${ }^{3}$, Hesham M Amin ${ }^{4}$ and Yupo $\mathrm{Ma}^{5}$ \\ ${ }^{1}$ Department of Laboratory Medicine and Pathology, Cross Cancer Institute and University of Alberta, \\ Edmonton, Alberta, Canada; ${ }^{2}$ Department of Medicine, Cross Cancer Institute and University of Alberta, \\ Edmonton, Alberta, Canada; ${ }^{3}$ Calgary Laboratory Service and University of Calgary, Calgary, Alberta, \\ Canada; ${ }^{4}$ Department of Hematopathology, University of Texas MD Anderson Cancer Center, Houston, \\ TX, USA and ${ }^{5}$ Department of Pathology, Nevada Cancer Center, Las Vegas, NV, USA
}

\begin{abstract}
Mantle cell lymphoma is an aggressive B-cell lymphoma for which the biology is incompletely understood. Previous studies have reported that somatic hypermutation of the variable region of the immunoglobulin heavy chain gene $\left(V_{H}\right)$, as commonly defined as $<98 \%$ homology, can be detected in approximately one-third of mantle cell lymphoma, although the $V_{H}$ mutation status has not been found to significantly correlate with patient survival. In this study, we assessed $V_{H}$ mutation in $\mathbf{5 5}$ mantle cell lymphomas using a method slightly different from those used in the previous studies, and we came to different conclusions. Using DNA extracted from formalin-fixed/paraffin-embedded tumors in all cases, we identified monoclonal IGH bands in 54 of 55 cases with the $F R 1 c / J_{H}$ primer; a monoclonal $I G H$ band was amplified using another $I G H$ primer set, $F R 256 / J_{H}$, in the remaining case. Cloning was performed in all cases, and an average of six clones were sequenced and analyzed for each case. Intraclonal heterogeneity was detected in $45(82 \%)$ cases. Further analysis was performed in 53 cases, in which a predominant IGH species was identified. Most ( 32 of 53 cases, $60 \%$ ) cases were 'mutated', with $<98 \%$ homology. $V_{H} 1-69, V_{H} 4-59$ and $V_{H} 3-74$ were utilized in $29(55 \%)$ cases. Intraclonal evolution and nonproductive $V_{H}$ rearrangements were more frequent in the mutated group. Patients with the 'mutated' genotype had longer overall survival $(P=\mathbf{0 . 0 1 7}$, Log rank) that is independent of the international prognostic index. To conclude, our data suggest that the $V_{H}$ mutation frequency in mantle cell lymphoma may be higher than previously believed. Importantly, using our methodology, we found that the $V_{H}$ mutation status may be a useful prognostic marker for these patients.
\end{abstract}

Modern Pathology (2006) 19, 1498-1505. doi:10.1038/modpathol.3800677; published online 15 September 2006

Keywords: mantle cell lymphoma; IGH somatic hypermutation; prognostic significance

Mantle cell lymphoma is a distinct clinicopathologic entity recognized by the World Health Organization Classification Scheme. ${ }^{1}$ The genetic hallmark of this disease is the chromosomal abnormality, the $\mathrm{t}(11 ; 14)(\mathrm{q} 13 ; \mathrm{q} 32)$, which results in cyclin D1 overexpression. ${ }^{2}$ Using transgenic mouse models, two research groups have shown that enforced cyclin D1 expression in B cells is not sufficient for lymphomagenesis. ${ }^{3,4}$ Recent studies, including a number of

Correspondence: Dr R Lai, MD, PhD, Department of Laboratory Medicine and Pathology, Cross Cancer Center and University of Alberta, 11560 University Avenue, Edmonton, Alberta, Canada T6G 1 Z2.

E-mail: Raymondl@cancerboard.ab.ca

Received 28 June 2006; revised 29 July 2006; accepted 31 July 2006; published online 15 September 2006 oligonucleotide array studies, revealed additional biochemical abnormalities in mantle cell lymphoma, such as defects in the apoptotic pathway, cell cycle progression and DNA repair. ${ }^{5-10}$ To further understand the biology of this type of B-cell neoplasm, one approach is to examine the somatic hypermutation of the variable region of the immunoglobulin heavy chain $\left(V_{H}\right)$ gene. In chronic lymphocytic leukemia, it has been shown that a subset of cases has relatively high levels of $V_{H}$ somatic mutation, and mutated cases were associated with better clinical outcome when using $<98 \%$ homology as the cutoff. ${ }^{11-14}$ More recently, several large studies were performed to examine the $V_{H}$ mutation status in mantle cell lymphoma; using $<98 \%$ homology (compared to the germline sequences) as the cutoff, it was found that $29-34 \%$ of 
mantle cell lymphoma have relatively high rates of $V_{H}$ mutation. ${ }^{15-18}$ In contrast to chronic lymphocytic leukemia, no strong correlation was identified between the degree of $V_{H}$ homology and patient survival, although one study revealed a significant correlation between the use of $V_{H} 3-21$ and a better clinical outcome. ${ }^{15}$ These studies also identified a bias in the utilization of specific $V_{H}$ gene segments in mantle cell lymphoma, with $V_{H} 3-21$ being the most frequently utilized region. These findings implicate a role of antigen stimulation in the lymphomagenesis of these tumors.

In this study, we assessed the $V_{H}$ somatic mutation status in a cohort of mantle cell lymphomas collected in one institution, using a slightly modified protocol described in a recent study, ${ }^{19}$ that is different from those used in most of the previous studies. First, in contrast with all of the previous studies examining $V_{H}$ mutations in mantle cell lymphoma, we employed FR1c/ $\mathrm{J}_{\mathrm{H}}$ to amplify the $I G H$ monoclonal species, as this primer set has been reported to be more sensitive than most of the $I G H$ primer sets employed in the previous studies. ${ }^{20}$ Second, we employed DNA extracted from paraffin-embedded tumors in all cases, whereas a mixture of DNA and RNA templates was used in most of the previous studies. Third, we performed cloning in all cases, whereas direct DNA sequencing was used in the vast majority of the cases in the previous studies. For cases with $<98 \%$ homology, we analyzed the predicted amino-acid sequence to determine the ratio of replacement mutations vs silent mutations, which allow us to determine if the pattern of $V_{H}$ mutations resembles that of memory/antigenselected B cells or that of germinal center B cells before substantially going through the antigen selection process. Our data revealed that $60 \%$ of our cases carried the $V_{H}$-mutated genotype using a cutoff of $<98 \%$ homology, and this frequency is higher than those reported previously. Although we also identified a bias of $V_{H}$ utilization in our cohort, some of the most commonly utilized $V_{H}$ regions in our cohort do not overlap with those identified in the previous studies. Importantly, we identified a significant correlation between the $V_{H}$-mutated genotype and longer overall survival.

\section{Materials and methods}

\section{Patients and Tumor Samples}

All mantle cell lymphoma cases initially diagnosed at the Cross Cancer Institute between 1993 and 2005 were identified, and a total of 55 cases with sufficient materials were included in this study. All tumors were formalin-fixed and paraffin-embedded, and all of the tissues used in this study have been histologically confirmed to be composed of mostly lymphomatous tissues. The diagnosis of all cases was made in accordance with World Health Organization Classification Scheme. ${ }^{1}$ By immunohistochemistry, all of the cases were positive for CD20, CD5/CD43, cyclin D1 and negative for CD23. All tumors were derived from solid organs and the vast majority (>90\%) of the cases were nodal diseases, with four cases diagnosed as the blastic variant. The diagnosis of all cases was confirmed by three hematopathologists (RL, HMA, YM). The study was reviewed and approved by the institutional ethics committee.

\section{DNA Extraction, Polymerase Chain Reaction and Cloning}

DNA was extracted from formalin-fixed, paraffinembedded tumors using the QIAamp DNA Mini kit (Qiagen, Mississauga, ON, Canada) according to the manufacturer's recommended protocol, except that paraffin was removed with three washes of HemoDe (Fisher Scientific, Pittsburg, PA, USA) instead of xylene. Polymerase chain reaction (PCR) was performed using $0.5 \mu \mathrm{g}$ of extracted tumor DNA, the HotStarTaq Master Mix Kit (Qiagen) and the FR1c primer set. ${ }^{20}$ If no monoclonal IGH bands were obtained, FR256 was used as the alternative IGH primer set. Forty PCR cycles were performed using an annealing temperature of $55^{\circ} \mathrm{C}$. Amplified monoclonal bands were detected on $8 \%$ polyacrylamide gels, excised and DNA was subsequently extracted from the gel using the QIAquick Gel Extraction Kit following the 'user-developed' protocol described online (www.qiagen.com). Cloning of the monoclonal IGH products was performed in all cases. Extracted DNA was ligated into the pGEM-T vector (Promega, Madison, WI, USA) and subsequently transformed into DH5 $\alpha$ cells (Invitrogen, Carlsbad, CA, USA). Five to 12 clones were chosen and inoculated into $10 \mathrm{ml}$ of laurier broth with $150 \mu \mathrm{g} / \mathrm{ml}$ of ampicillin overnight with shaking at $37^{\circ} \mathrm{C}$. The cloned plasmids were extracted using the QIAprep Spin Miniprep Kit (Qiagen). DNA sequencing was performed using the BigDye Terminators (ABI3100, San Jose, CA, USA).

\section{Analysis of the IGH Sequences}

The DNA sequence from each clone was aligned to the immunoglobulin gene sequence database (www.ncbi.nlm.nih.gov/igblast). The germline $V_{H}$ region possessing the highest homology to the DNA sequence being analyzed was regarded as the $V_{H}$ region utilized. Each individual clone was given a degree of homology compared to its most closely related specific germline $V_{H}$ region, and the degree of homology was expressed as a percentage. For each case, the most frequently utilized $V_{H}$ gene segment was designated as the predominant $V_{H}$ species, and an average of the homology percentages for all the clones belonging to the predominant 
species was determined. Assessment of intraclonal evolution was based on the DNA sequences of $\geq 3$ clones, in which stepwise increases in the number of mutations were observed, as suggested previously. ${ }^{21}$ Data from $>50 \%$ of our cases were generated by two individuals performing experiments at different times, and their results correlated in all cases. In addition, the vast majority of the mutations in each case were consistent among all of the predominant clones, and thus, these mutations were not generated as a result of Taq errors.

\section{Analysis of $V_{H}$ Somatic Mutations}

Excluding clones that were non-productive (as defined as point mutations or frameshifts that result in premature stop codons), the amino-acid sequences for all clones were determined using the ChromasPro sequence analysis software (Technelysium Pty Ltd, Tewantin, Australia). As described previously, ${ }^{19}$ we assessed changes in the $I G H$ sequence compared to the germline using two methods established for B-cell lymphomas, ${ }^{22,23}$ on the basis that non-antigen-selected B-cells are expected to preferentially possess mutations leading to replacement of amino acids (ie replacement mutations) over silent mutations in the complementary determining regions. In contrast, memory- or antigen-selected B-cells will be expected to preferentially possess silent mutations over replacement mutations in the framework regions, to minimize dramatic alterations to the immunoglobulin structure. One method (accessible at www-stat.standford.edu/immunoglobulin) gives the probability of the observed mutation pattern that is generated by chance only; thus, a $P$-value of $<0.05$ is regarded as significant and strongly indicative of an antigenselected ('memory B-cell') genotype. The other method calculates the ratio of the sum of replacement mutations to the sum of silent mutations (R/S ratio) in the framework regions, and an R/S ratio of $<1.1$ correlates with an antigen-selected or memory B-cell genotype whereas an R/S ratio of $>1.9$ correlates with a genotype seen in non-antigenselected, germinal center B-cells. ${ }^{22}$ The presence or absence of clones with non-productive $I G H$ was also documented for each case.

\section{Statistical Analysis}

Various clinical parameters were compared between cases with relatively low levels of homology (ie $<98 \%$ ) vs cases with relatively high levels of homology (ie $\geq 98 \%$ ) using Fisher's exact test or the Spearman rank correlation test. Survival data were calculated from the date of initial diagnosis until the last follow-up or death. Kaplan-Meier survival analysis and log-rank analysis were performed to assess the prognostic values of various parameters in our series. Multivariate analysis using the Cox proportional hazards model was performed to assess the significance of the somatic mutation status as predictors of survival while adjusting for the other clinical parameters with hazard ratios with $95 \%$ confidence limits. The $P$-value was calculated as two-sided.

\section{Results}

\section{IGH Rearrangements and $V_{H}$ Somatic Hypermutation}

Using the primer set FR1c $/ \mathrm{J}_{\mathrm{H}}, 54$ of 55 cases show amplifiable monoclonal IGH bands. Amplifiable PCR product was obtained in the remaining one case using the $I G H$ primer set FR256/ $/ \mathrm{J}_{\mathrm{H}}$. On average, six clones were sequenced and analyzed for each case. Of these 55 cases, a predominant species was identified in 53 cases; the remaining two cases were composed of a mixture of single clones of different $V_{H}$ gene segments. Of the 53 cases that had a predominant $V_{H}$ clone, $32(60 \%)$ cases had $<98 \%$ homology and 21 (40\%) cases had $\geq 98 \%$ homology, based on the average homology for the predominant clones. For those 32 cases with the mutated genotype, 12 had $<96 \%$ homology, 8 had $\geq 96$ to $97 \%$ and 12 had $\geq 97$ to $<98 \%$.

We identified an obvious bias in the utilization of specific $V_{H}$ regions in the predominant clones of these 53 cases, with $V_{H} 1-69$ (11 of 53 cases, $21 \%$ ), $V_{H} 4-59\left(10\right.$ of 53 cases, $19 \%$ ) and $V_{H} 3-74$ (eight of 53 cases, 15\%) being the most frequently used. As shown in Table 1, the use of these $V_{H}$ regions was not random, and in fact correlated with the degree of $V_{H}$ homology. Notably, all 10 cases positive for $V_{H} 4$ 59 had $\geq 98 \%$ homology, whereas all eight cases positive for $V_{H} 3-74$ had $<98 \%$ homology. All 11

Table 1 Correlation between the degree of $V_{H}$ homology and the utilization of specific $V_{H}$ regions in 53 mantle cell lymphomas

\begin{tabular}{|c|c|c|c|c|}
\hline Degree of homology & $<96 \%$ & $\geq 96$ to $<97 \%$ & $\geq 97$ to $<98 \%$ & $\geq 98$ to $100 \%$ \\
\hline$V_{H} 1-69(11$ cases, $21 \%)$ & 0 & 0 & 5 & 6 \\
\hline$V_{H} 4-59(10$ cases, $19 \%)$ & 0 & 0 & 0 & 10 \\
\hline$V_{H} 3-74(8$ cases, $15 \%)$ & 0 & 5 & 3 & 0 \\
\hline$V_{H} 3-34(3$ cases, $6 \%)$ & 1 & 1 & 1 & 0 \\
\hline Other $V_{H}$ regions & 11 & 2 & 3 & 5 \\
\hline Total cases for each category of $V_{H}$ homology & 12 & 8 & 12 & 21 \\
\hline$\%$ of the total cases & \multicolumn{2}{|c|}{$60 \%$ (32 of 53 cases) } & \multicolumn{2}{|c|}{$40 \%$ ( 21 of 53 cases) } \\
\hline
\end{tabular}


cases positive for $V_{H} 1-69$ had $\geq 97 \%$ homology. One of the less frequently utilized $V_{H}$ regions, $V_{H} 3-34$, was also found only in cases with $<98 \%$ homology.

To further characterize the nature of the $V_{H}$ mutations identified in these 53 cases, we determined the R/S ratio for the predominant species from each case. As described in Materials and Methods, an R/S ratio of $<1.1$ is associated with memory- or antigen-selected B cells whereas an R/S ratio of $>1.9$ is associated with germinal center $B$ cells without substantially going through antigen selection. As illustrated in Figure 1, clones with an R/S ratio of $>1.9$ were found predominantly in cases with relatively high levels of homology. For instance, none of the case with clones carrying $<95 \%$ homology and only $20 \%$ of cases with $95 \%$ to $<96 \%$ homology had clones carrying an R/S ratio of $>1.9$. Up to $92 \%$ of cases with 96 to $<98 \%$ homology contained clones that had an R/S ratio of

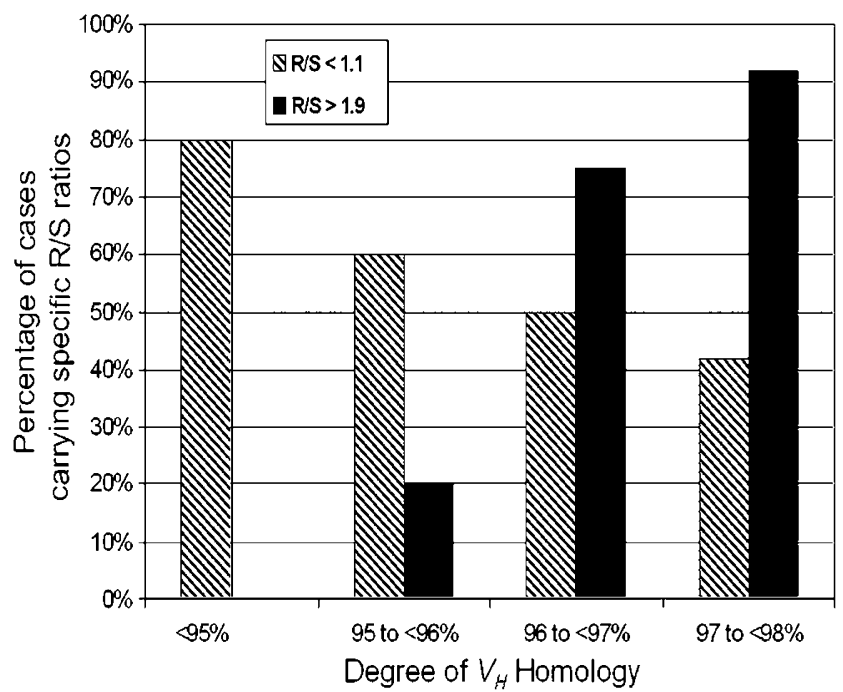

Figure 1 The histogram summarized the percentage of cases within each $V_{H}$ mutational category that carried clones with an $\mathrm{R} / \mathrm{S}$ ratio of $<1.1$ or $>1.9$. There is a trend for increasing frequency of cases carrying an R/S ratio of $>1.9$ with increasing the degree of homology, and vice versa for cases carrying clones with an $\mathrm{R} / \mathrm{S}$ ratio of $<1.1$.
$>$ 1.9. Conversely, cases carrying clones with an R/S ratio of $<1.1$ were most frequently found in those with $<95 \%$ homology, and the frequency of cases carrying clones with an R/S ratio of $<1.1$ decreased gradually with increasing degrees of homology, up to $<98 \%$. Using the alternative method published by Lossos et al, ${ }^{23} 11$ of 32 mutated cases had a $P$ value of $<0.05$ whereas none of the 21 unmutated cases had a $P$-value of $<0.05$, and this difference is statistically significant (0.002, Fisher's exact test) (Table 2).

As illustrated in Table 2, non-productive IGH rearrangements involving $\geq 1$ clone were detectable in 26 of $53(49 \%)$ cases overall, with 22 of $32(71 \%)$ cases carrying $<98 \%$ homology and four of 21 $(19 \%)$ cases carrying $\geq 98 \%$ homology; the difference between the two groups of mantle cell lymphoma is statistically significant $(P=0.0007$, Fisher's exact test). Most of the non-productive IGH rearrangements were due to creation of premature stop codons, large deletions or insertions. Intraclonal evolution was identified in nine of 46 $(20 \%)$ cases, with seven of $28(25 \%)$ cases carrying $<98 \%$ homology and two of $18(11 \%)$ cases carrying $\geq 98 \%$ homology; the difference between the two groups of tumors is not statistically significant $(P=0.45$, Fisher's exact test $)$.

\section{Clinical Data}

Of the 53 patients studied in detail, 47 were men and six were women, with a median age of 64 years (range, 41-88 years). At follow-up, 30 patients had died and 23 were alive. Most $(89 \%)$ patients were treated with CHOP (cyclophosphamide, doxorubicin, vincristine and prednisone) or chlorambucil alone. The median follow-up for those who were alive at the last follow-up was 24 months (range, 2-63 months). The median time to death was 13.5 months (range, 1-83 months). Other data regarding the clinical stage, international prognostic index, splenomegaly, leukemic disease (defined by $>5 \times 10^{9} / 1$ absolute lymphocyte count) are illustrated in Table 3 . No significant correlation was identified between the degree of homology

Table 2 Comparison of the $V_{H}$ mutations in mantle cell lymphoma with $<98$ or $\geq 98 \%$ homology

\begin{tabular}{ccc}
\multicolumn{2}{c}{ Degree of homology } & \multirow{2}{*}{ P-value } \\
\cline { 1 - 1 } & $\geq 98 \%$ to $100 \%$ & \\
\hline 22 of 32 cases, $71 \%$ & 4 of 21 cases, $19 \%$ & 0.0007 \\
7 of 28 cases, $25 \%$ & 2 of 18 cases, $11 \%$ & 0.45 \\
11 of 32 cases, $34 \%$ & 0 of 21 cases & 0.002 \\
\hline
\end{tabular}

Non-productive clone $(n=53)$

Intraclonal evolution $(n=46)$

Stanford method ${ }^{\text {a }}$ (cases with a $P$-value $<0.05$ )
7 of 28 cases, $25 \%$

${ }^{\mathrm{a}}$ The Stanford method (www-stat.standford.edu/immunoglobulin), as described in the Materials and methods, gives the probability of the observed mutation pattern in the framework regions and complementary determining regions that is generated by chance only; thus, a $P$-value of $<0.05$ is regarded as significant and strongly indicative of an antigen-selected ('memory B-cell') genotype. 
Table 3 Clinical features of 53 mantle cell lymphoma patients and their correlation with the $V_{H}$ mutation status

\begin{tabular}{|c|c|c|c|}
\hline & \multicolumn{2}{|c|}{ Degree of homology } & \multirow[t]{2}{*}{ P-value } \\
\hline & $<98 \%$ & $\geq 98$ to $100 \%$ & \\
\hline Median age $(n=53)$ & 65.5 years & 64.0 years & NS \\
\hline Clinical stage III/IV $(n=51)$ & 26 of 31 cases, $84 \%$ & 19 of 20 cases, $95 \%$ & 0.38 \\
\hline IPI score $(\geq 3)(n=42)$ & 8 of 25 cases, $32 \%$ & 9 of 17 cases, $53 \%$ & 0.21 \\
\hline Leukemic disease $^{\mathrm{a}}(n=51)$ & 3 of 31 cases, $10 \%$ & 4 of 20 cases, $20 \%$ & 0.41 \\
\hline Splenomegaly $(n=48)$ & 6 of 28 cases, $21 \%$ & 8 of 20 cases, $40 \%$ & 0.21 \\
\hline Performance status $(\geq 1)(n=48)$ & 6 of 30 cases, $20 \%$ & 5 of 18 cases, $28 \%$ & 0.72 \\
\hline B symptoms $(n=47)$ & 6 of 27 cases, $22 \%$ & 8 of 20 cases, $40 \%$ & 0.21 \\
\hline Gender & $\mathrm{M}=29, \mathrm{~F}=3$ & $\mathrm{M}=18, \mathrm{~F}=3$ & 0.67 \\
\hline
\end{tabular}

F, female; IPI, international prognostic index; M, male; NS, nonsignificant.

${ }^{\mathrm{a}}$ Leukemic disease was defined as $>5 \times 10^{9} / \mathrm{l}$ in the absolute white cell count.

(categorized based on $<98$ or $\geq 98 \%$ ) and these clinical parameters. We also had four cases with blastoid morphologic features in our series, but no significant correlation was found between these blastic cases and the degree of homology, probably owing to the small number of this morphologic variant. Interestingly, if a degree of homology of $\geq 97 \%$ (instead of $98 \%$ ) was used as the cutoff, we found a significant correlation between the presence of leukemic involvement and a high degree of homology, as none of the 20 cases with $<97 \%$ homology had leukemic involvement, compared to seven of 31 cases with $\geq 97 \%$ homology $(P=0.034$, Fisher's exact test).

\section{Correlation between Clinical Parameters and the $V_{H}$ Mutation Status}

Using Fisher's exact test, categorical comparison was also performed using various clinical parameters, and the results are summarized in Table 3. The prognostic value of $I G H$ mutation in mantle cell lymphoma was assessed using the log-rank test, and the results are illustrated in Figure 2. Cases with $\geq 98 \%$ homology had a significantly shorter overall survival than those with $<98 \%$ homology $(P=0.017)$. The use of different degrees of homology as the cutoff yielded no statistically significant correlation between the $V_{H}$ mutation status and the overall survival. Using univariate analysis, we also examined the prognostic values of other clinical parameters including patient age $(<60$ years $v s \geq 60$ years at the time of initial diagnosis) $(P=0.40)$, clinical stage (stage $0-$ II vs stage III-IV) $(P=0.80)$ and international prognostic index $(0-2$ vs 3-5) $(P=0.26)$. Only the performance status was predictive of survival $(P=0.021, \log$ rank). Lactate dehydrogenase level marginally correlated with survival $(P=0.052)$. Using multivariable analysis (Cox proportional hazards analysis), the prognostic value of $V_{H}$ mutation status was independent of performance status and the international prognostic index, as summarized in Table 4.

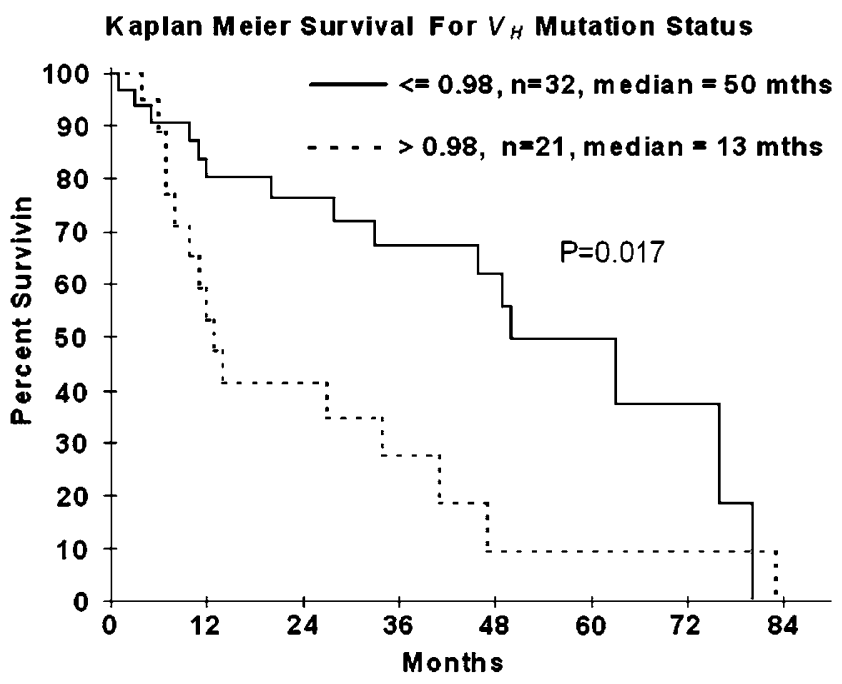

Figure 2 Kaplan-Meier survival analysis for 53 mantle cell lymphoma patients, with a comparison between those with $\geq 98 \%$ homology vs those with $<98 \%$ homology.

Table 4 Multivariable analysis of survival incorporating the $V_{H}$ mutation status performance status and international prognostic index

\begin{tabular}{lcc}
\hline & Hazard ratio & P-value \\
\hline Mutated vs un-mutated & 3.71 & 0.01 \\
Performance status $(<1$ vs $\geq 1)$ & 1.64 & 0.45 \\
International prognostic index & 1.05 & 0.93 \\
\hline
\end{tabular}

\section{Discussion}

Although the $t(11 ; 14)(q 13 ; q 23)$ chromosomal abnormality and cyclin D1 overexpression are consistent findings in mantle cell lymphoma, more recently published data suggest that the pathogenesis of this disease likely involves defects of multiple cellular pathways which are incompletely understood. Analysis of $V_{H}$ mutation has been shown to yield important insights into the biology 
of B-cell neoplasms. As $V_{H}$ mutations normally occur in the germinal centers and this process is dependent on antigen stimulation and T-cell contribution, detection of the $V_{H}$-mutated genotype in a B-cell neoplasm implicates a significant role of antigenic stimulation during lymphomagenesis. On the other hand, the finding of the unmutated $V_{H}$ genotype in a B-cell neoplasm may suggest a relative lack of antigenic stimulation during lymphomagenesis (ie antigen-inexperienced $\mathrm{B}$ cells), or the existence of biochemical defects leading to an impairment of the $V_{H}$ somatic hypermutation process.

In mantle cell lymphoma, several previous studies have demonstrated that a relatively high rate of $V_{H}$ mutations, commonly defined as $<98 \%$ compared to the $V_{H}$ germline sequences, is detectable in up to one-third of the cases. In our study, we also found that a subset of mantle cell lymphoma had the mutated genotype, although the frequency of $V_{H}$ mutation is substantially higher in our study (ie $60 \%$ ). This discrepancy is likely multifactorial, although we believe that differences in the methodology contribute greatly to this discrepancy. First, cloning was not performed in most of the cases evaluated in the previous studies, presumably because RNA templates were used. As there is clonal heterogeneity in mantle cell lymphoma, as revealed by a previous study ${ }^{16}$ and our current study, the cell clone analyzed may not be the representative or predominant $I G H$ species. Based on our experience from this study, the lack of cloning may lead to significantly different results. As pointed out by Camacho et al, ${ }^{16}$ the presence of clonal heterogeneity may be secondary to the failure of allelic exclusion, as reported in chronic lymphocytic leukemia. ${ }^{24}$ Second, DNA templates were used uniformly in all cases in our study, whereas a mixture of RNA and DNA templates were used in most of the previous studies. ${ }^{15,17,18}$ We believe that results from the use of RNA templates may yield different results from the use of DNA templates, as different rearranged $I G H$ species may be transcribed at different rates and thus they may be represented differently at the RNA level. To this point, we have recently performed parallel studies using RNA template as well as DNA templates from 12 cases of mantle cell lymphoma, and our results are in keeping with this concept (manuscript in preparation). Third, the use of different $I G H$ primers may contribute to the discrepancies between the results of our study and those published previously. In our study, we primarily employed FR1c, which has been reported to be more sensitive than the other commonly used IGH primer sets. ${ }^{20}$ In keeping with this concept, at least one of the previous studies reported that successful amplification of the monoclonal IGH gene was found in only 65 of 80 cases evaluated, ${ }^{17}$ whereas our study successfully amplified all but one case. We also have considered the possibility that different $I G H$ primers may have bias toward amplifying different $V_{H}$ regions. Lastly, all of the four previous large studies were from European centers, ${ }^{15-18}$ and we cannot exclude the possibility that the North American population (as in this study) may have different characteristics than their European counterparts.

Similar to the previous studies, we identified a strong bias for specific $V_{H}$ utilization in mantle cell lymphoma. These three $V_{H}$ regions were utilized in $55 \%$ of the cases examined. In the study by Camacho et $a l,{ }^{16}$ five $V_{H}$ regions, including $V_{H} 3-21$, $V_{H} 4-34, V_{H} 3-23, V_{H} 4-59$ and $V_{H} 4-39$, account for $47 \%$ of their cases. Similar findings were reported by other studies, with $V_{H} 3-21, V_{H} 4-59$ and $V_{H} 4-34$ being most commonly implicated. ${ }^{15,18}$ In our study, we also identified a strong bias for $V_{H} 4-59$, but we identified $V_{H} 1-69$ and $V_{H} 3-74$ as the most frequent utilized $V_{H}$ regions, neither of which were highly represented in the previous studies. These discrepancies may be attributed to methodological differences, as described above. Alternatively, as discussed above, it is also possible that the differences in the $V_{H}$ utilization are related to variations in the pattern of antigenic stimulation between the North American patients (as in the current study) and the European patients (in the previous studies). Of note, other than $V_{H} 4-59$, the two most frequently utilized $V_{H}$ regions in the European studies (namely $V_{H} 3-21$ and $V_{H} 4-34$ ) were identified in our study, although their frequencies were relatively low (2 and $6 \%$, respectively). We also identified that various $V_{H}$ regions correlate with the degree of homology. As summarized in Table 1, $V_{H} 4-59$ was restricted to the unmutated cases. At least one other report describes similar findings. ${ }^{16}$

We detected a significant correlation between the degree of $V_{H}$ homology and overall survival. Using $<98 \%$ homology as the cutoff, mutated cases had a significantly longer overall survival. Multivariate analysis showed that the prognostic value of $V_{H}$ mutation was independent of the patient age, performance status, international prognostic index and clinical stage. Thus, this finding is analogous to that of chronic lymphocytic leukemia. ${ }^{14}$ Interestingly, $<98 \%$ was found to be the only cutoff that resulted in a significant correlation between survival and the $V_{H}$ mutation status.

Our findings support the concept that antigen stimulation most likely plays a role in the development of mantle cell lymphoma. Evidence to support this concept came from the finding of a relatively high rate of $V_{H}$ mutations in a subset of these tumors. Analysis of ongoing intraclonal evolution and the presence/absence of non-productive IGH has provided additional evidence. We identified nine of $46(20 \%)$ cases carrying evidence of intraclonal evolution, including two of 18 cases $(11 \%)$ in the unmutated group. Whereas other studies did not come to the same conclusions, cloning was not performed in most of these studies and thus, the presence/absence of intraclonal evolu- 
tion simply cannot be assessed. At least one study reported similar observations. ${ }^{25}$ With regards to our method of interpretating intraclonal evolution, we required at least three clones showing stepwise increment of mutations, as described previously. Thus, it is highly unlikely that these changes are due to Taq error.

The findings of both intraclonal evolution and non-productive IGH rearrangements in the unmutated group are rather surprising to us. These findings raise the possibility that a subset of the unmutated cases may not be antigen-inexperienced, as creation of non-productive $I G H$ rearrangement and intraclonal evolution are features of germinal center B cells that are dependent on antigen stimulation. Thus, many mantle cell lymphomas may in fact be derived from the germinal centers, rather than the mantle zones. Based on these findings, we speculate that antigen stimulation occurs during the lymphomagenesis in at least a proportion of these highly homologous cases, but the reason for their lack of mutated phenotype may be related to defective machinery responsible for somatic hypermutation. In this regard, activationinduced cytidine deaminase has been shown to be important in mediating somatic hypermutation. ${ }^{26,27}$ In chronic lymphocytic leukemia, there is evidence that cases with high IGH homology express activation-induced cytidine deaminase differently from cases with relatively low homology $(<98 \%),{ }^{28}$ and it has been suggested that this enzyme is defective in a subset of the highly homologous cases and this biochemical defect accounts for their unmutated phenotype. Similar defects may occur in mantle cell lymphoma and these defects may account for the relative lack of $V_{H}$ somatic mutation in a subset of unmutated cases. A recent paper has shown data related to the expression of activation-induced cytidine deaminase in mantle cell lymphoma; although the expression pattern of this enzyme is variable among these tumors, there was no significant correlation between the pattern of expression and the degree of $V_{H}$ homology. ${ }^{29}$ Nevertheless, the number of cases included in this study was relatively small, and further studies are probably needed to clarify this issue.

\section{Acknowledgements}

This study is supported in part by research grants from the Alberta Cancer Board and the Canadian Cancer Society awarded to RL.

\section{References}

1 Swerdlow SH, Berger F, Isaacson PI, et al. Mantle cell lymphoma. In: Jaffe ES, Harris NL, Stein H, Vardiman JW (eds). World Health Organization Classification of Tumors. Pathology and Genetics of Tumors of Haema- topoietic and Lymphoid Tissues. IARC Press: Lyon, France, 2001, pp 168-170.

2 Campo E, Raffeld M, Jaffe ES. Mantle-cell lymphoma. Semin Hematol 1999;36:115-127.

3 Bodrug SE, Warner BJ, Bath ML, et al. Cyclin D1 transgene impedes lymphocyte maturation and collaborates in lymphomagenesis with the myc gene. EMBO J 1994;13:2124-2130.

4 Lovec H, Grzeschiczek A, Kowalski MB. Cyclin D1/bcl1 cooperates with myc genes in the generation of B-cell lymphoma in transgenic mice. EMBO J 1994;13:34873495.

5 Schaffner C, Idler I, Stilgenbauer S, et al. Mantle cell lymphoma is characterized by inactivation of the ATM gene. Proc Natl Acad Sci USA 2000;97:2773-2778.

6 Hernandez L, Fest T, Cazorla M, et al. p53 gene mutations and protein overexpression are associated with aggressive variants of mantle cell lymphomas. Blood 1996;87:3351-3359.

7 Pinyol M, Hernandez L, Cazorla M, et al. Deletions and loss of expression of $\mathrm{P} 16^{\text {INK4a }}$ and $\mathrm{P} 21^{\text {Waf-1 }}$ genes are associated with aggressive variants of mantel cell lymphomas. Blood 1997;89:272-280.

8 Martinez N, Camacho FI, Algara P, et al. The molecular signature of mantle cell lymphoma reveals multiple signals favoring cell survival. Cancer Res 2003;63: 8226-8232.

9 Rosenwald A, Wright G, Wiestner A, et al. The proliferation gene expression signature is a quantitative integrator of oncogenic events that predicts survival in mantle cell lymphoma. Cancer Cell 2003; 3:185-197.

10 Hofmann WK, de Vos S, Tsukasaki K, et al. Altered apoptosis pathways in mantle cell lymphoma detected by oligonucleotide microarray. Blood 2001;98:787-794.

11 Hamblin TJ, Davis Z, Gardiner A, et al. Unmutated $\operatorname{IgV}(\mathrm{H})$ genes are associated with a more aggressive form of chronic lymphocytic leukemia. Blood 1999;94:1848-1854.

12 Maloum K, Davi F, Merle-Beral H, et al. Expression of unmutated $V_{H}$ genes is a detrimental prognostic factor in chronic lymphocytic leukemia. Blood 2000;96: 377-379.

13 Krober A, Seiler T, Benner A, et al. V(H) mutation status, CD38 expression level, genomic aberrations, and survival in chronic lymphocytic leukemia. Blood 2002;100:1410-1416.

14 Damle RN, Wasil T, Fais F, et al. Ig V gene mutation status and CD38 expression as novel prognostic indicators in chronic lymphocytic leukemia. Blood 1999;94:1840-1847.

15 Walsh SH, Thorselius M, Johnson A, et al. Mutated VH genes and preferential VH3-21 use define new subsets of mantle cell lymphoma. Blood 2003;101:4047-4054.

16 Camacho FI, Algara P, Rodriguez A, et al. Molecular heterogeneity in mantle cell lymphoma defined by the use of specific $V_{H}$ gene and the frequency of somatic mutations. Blood 2003;101:4042-4046.

17 Orchard J, Garand R, Davis G, et al. A subset of $\mathrm{t}(11 ; 14)$ lymphoma with mantle cell features displays mutated $\operatorname{Ig} V_{H}$ genes and includes patients with good prognosis, nonnodal disease. Blood 2003;101:4975-4981.

18 Kienle D, Krober A, Katzenberger T, et al. $V_{H}$ mutation status and VDJ rearrangement structure in mantle cell lymphoma: correlation with genomic aberrations, clinical characteristics, and outcome. Blood 2003; 102:3003-3009. 
19 Timms JM, Bell A, Flavell JR, et al. Target cells of Epstein-Barr-virus (EBV)-positive post-transplant lymphoproliferative disease: similarities to ENV-positive Hodgkin's lymphoma. Lancet 2003;361:217-223.

20 Aubin J, Davi F, Nguyen-Salomon F, et al. Description of a novel FR1 IgH PCR strategy and its comparison with three other strategies for the detection of clonality in B cell malignancies. Leukemia 1995;9:471-479.

21 Cleary ML, Galili N, Trela M, et al. Single cell origin of bigenotypic and biphenotypic B cell proliferations in human follicular lymphomas. J Exp Med 1988;167: 582-597.

22 Klein U, Goossens T, Fischer M, et al. Somatic hypermutation in normal and transformed human $\mathrm{B}$ cells. Immunol Rev 1998;162:261-280.

23 Lossos IS, Tibshirani R, Narasimhan B, et al. The interference of antigen selection on Ig genes. J Immunol 2000;165:5122-5126.

24 Rassenti LZ, Kipps TJ. Lack of allelic exclusion in B cell chronic lymphocytic leukemia. J Exp Med 1997; 185:1435-1445.
$25 \mathrm{Du}$ MQ, Diss TC, Xu CF, et al. Ongoing immunoglobulin gene mutations in mantle cell lymphomas. Br J Haematol 1997;96:124-131.

26 Muramatsu M, Kinoshita K, Fagarasan S, et al. Class switch recombination and hypermutation require activation-induced cytidine deaminase (AID), a potential RNA editing enzyme. Cell 2000;102: $553-563$.

27 Martin A, Bardwell PD, Woo CJ, et al. Activationinduced cytidine deaminase turns on somatic hypermutation in hydridomas. Nature 2002;415: 802-806.

28 McCarthy H, Wierda WG, Barron LL, et al. High expression of activation-induced cytidine deaminase (AID) and splice variants is a distinctive feature of poor-prognosis chronic lymphocytic leukemia. Blood 2003;101:4903-4908.

29 Babbage G, Garand R, Robillard N, et al. Mantle cell lymphoma with $\mathrm{t}(11 ; 14)$ and unmutated or mutated $V_{H}$ genes expresses $A I D$ and undergo isotype switch events. Blood 2004;103:2795-2798. 